is known about postnatal CMV (pCMV) infection. Although pCMV infection in term healthy infant is mostly asymptomatic, serious gastrointestinal symptoms (vomiting, diarrhea, abdominal distension, hepatosplenomegaly, blood stools) are described in literature.

We describe two cases of infant hematemesis, focusing on the challenging differential diagnosis between pCMV gastritis and non IgE-mediated Cow's Milk Protein Allergy (CMPA) enteropathy.

Case 1: a 3-month-old female infant presented with growth impairment, hematemesis and melena. Blood and stool analysis (bacterial, viral and parasites panels) resulted normal. Cow's milk specific-IgE were negative.

Viral serologies revealed recent CMV infection with positive CMV-DNA Polimerase Chain Reaction (PCR) on urine and blood samples. Congenital CMV infection was ruled out through negative CMV-DNA PCR on the first day of life saliva sample. Esophagogastroduodenoscopy (EGD) revealed petechial elements in antral and duodenal-bulb mucosa; at biopsies normal eosinophils count and negative morphological research of Helicobacter pylori (HP) were found. Intranuclear CMV inclusion bodies were not detected and CMV immunostaining was negative.

Case 2: a 2-month-old male infant presented with dehydration, bloody diarrhoea, vomiting and feeding refusal. Blood analysis revealed severe hypoalbuminemia, anaemia and hypertransaminasemia. Stool examinations (bacterial, viral and parasites panels) and Mycobacterium Tuberculosis screening were negative. Allergological and immunological investigations resulted normal. CMV-DNA PCR on urine, blood and maternal milk samples were positive. CMV-DNA PCR on Guthrie card was negative. EGD and rettosigmoidoscopy revealed exudative active inflammation in duodenal mucosa. HP research was negative while CMV immunostaining visualized duodenal cells viral inclusions.

Discussion Paediatric hematemesis is mainly caused by foreign bodies ingestion, CMPA, infectious gastritis (Helicobacter pylori, CMV, parasites), drug-induced gastritis (steroids and FANS) and eosinophilic gastropathy. In our cases the differential diagnosis focused on pCMV infection and non IgE-mediated CMPA. Both infants had a partial clinical improvement after starting a cow's milk protein free diet. However, due to the concomitant pCMV infection and the absence of cow's milk specific-IgE, a definitive diagnosis could not be established.

In conclusion, paediatric hematemesis differential diagnosis turns out particularly challenging when considering non IgEmediated CMPA and pCMV gastropathy. In fact, neither the absence of cow's milk specific-IgE

\section{GLUTEN-DEGRADING MICROORGANISMS IN ADOLESCENTS WITH COELIAC DISEASE}

Andraž Kukovičič ${ }^{*}$, Tilen Seničar, Jernej Dolinšek, Maja Rupnik. Faculty of medicine University of Maribor

10.1136/archdischild-2021-europaediatrics.262

Oral and gut microbiota play an important role in the pathogenesis of coeliac disease. The aim of our study was isolation and comparison of gluten-degrading microorganisms (GDM) from feces and saliva of adolescent patients with coeliac disease (CD) and healthy controls (HC). Additionally, we compared genomes of the same bacterial species isolated from samples of feces and saliva obtained from the same individual.

Feces and saliva were obtained from $5 \mathrm{CD}$ patients (2 female, 3 male) on gluten-free diet (GFD) and 5 HC (3 female, 2 male) aged 13-18 years. Samples were inoculated on culturing medium (MCG3) with gluten as a major source of carbon and nitrogen. All colonies with lysis zone were further isolated in pure culture and identified using MALDI Biotyper (Bruker Daltonics). In 4 samples (3 CD, $1 \mathrm{HC}$ ), Whole genome sequencing (WGS) was performed on MiSeq platform (Illumina) on all strains that belonged to the same species and were isolated from fecal sample and from saliva in the same individual.

In the CD group 10 GDM strains were isolated (5 were not identified): 2 from feces and 8 from saliva. In contrast to the HC group, where 16 GDM were isolated (1 was not identified): 7 from feces and 10 from saliva, 1 GDM was isolated from both samples (saliva and feces). GDM isolated from $\mathrm{CD}$ samples belong to 3 genera of bacteria and 1 yeast (Candida albicans). The latter was also isolated in the HC group along with bacteria from 12 different genera. That indicates higher GDM diversity in $\mathrm{HC}$ compared with the $\mathrm{CD}$ group.

Three bacterial species were isolated from feces and saliva of the same individual: Veillonella parvula, Lactobacillus paracasei, Lactobacillus rhamnosus. WGS showed identical genomes only in L. rhamnosus. That could indicate transmission between oral cavity and gut.

We found that cultivable GDM are diverse and more often present in feces and saliva of $\mathrm{HC}$ than $\mathrm{CD}$, which could be the effect of GFD the CD patients were on. Genomically identical lactobacilli were detected in saliva and in feces of the same individual.

\section{HELICOBACTER PYLORI INFECTION IN CHILDREN WITH CELIAC DISEASE}

Ekaterina Orlova*, Novikova Valeria, Shapovalova Natalia, Gurina Olga, Dementieva Elena, Klikunova Ksenia. Federal State budgetary Institute of Higher Education «Saint-Petersburg State Pediatric Medical Universityy of the Ministry of Health of the Russian Federation

\subsection{6/archdischild-2021-europaediatrics.263}

Aim: to reveal the effect of $\mathrm{H}$. pylori on course of celiac disease (CD) in children.

Methods 58 children with histologically confirmed CG and newly diagnosed CD were examined. Children were divided into two groups according to presence of $\mathrm{H}$. pylori infection: the first group-12 H.pylori-positive and the second group 46 H.pylori-negative subjects. All patients underwent histological examination of gastric and duodenal biopsies, histological verification of $H$. pylori infection and biopsy urease test. Tissue transglutaminase antibodies (tTG IgA, IgG) anti- $\mathrm{H}+/ \mathrm{K}+$ ATPase and anti-intrinsic antibodies, were measured by ELISA. Results Mean age of patients was $11.33 \pm 3.06$ years in group 1 and $10.38 \pm 1.43$ years in group $2(p=0.582)$. Manifestation of CD didn't differ statistically significantly in groups. 